(3) Exhaustion of the colonic musculature.

(4) Disturbance of the defaecation reflexes.

In normal adults the most common causes are those relating to the overactivity of the segmenting movements of the distal colon but a small number of persons have a true atonic constipation. In paraplegic patients, particularly those with lesions of the lumbar sacral cord, there is not only disturbance of the defaecation reflexes but probably hypersegmentation as well.

\title{
REFERENCES
}

BARClay, A. E. (1935). Brit. F. Radiol. 8, 625.

Chaudhary, N. A. \& Truelove, S. C. (I96I). Gastroenterology, 40, I.

ConNell, A. M. (1961a). Gut, 2, 175.

Connell, A. M. (196Ib). Proc. Roy. Soc. Med. 54, I2.

ConNell, A. M. (1962). Gut. 3, 342.

Connell, A. M., Frankel, H. \& Guttmann, L. (1963). Paraplegia, I, 98.

Connell, A. M., Lennard Jones, J. E. \& Madanagopalan, N. (1964). Proc. Roy. Soc. Med. 57, 894 .

Connell, A. M., Hilton, C., Irvine, G., Lennard Jones, J. E. \& Misiewicz, J. J. (i965). Brit. med. F. 2, 1095.

DelleR, D. J. \& WANGEL, A. G. (I965). Gastroenterology, 48, 45.

HANSKy, J. \& ConNell, A. M. (1962). Gut, 3, 187.

HerTz, A. F. (1907). Guy's Hosp. Rep. 6r, 389.

Keele, C. A. \& Neil, E. (1961). Samson Wright's Applied Physiology. London: Oxford University Press.

Ritchie, J. A., Ardran, G. M. \& Truelove, S. C. (I962). Gastroenterology, 43, 643.

Spriggs, E. A., Code, C. F., Bargen, J. A., Curtiss, R. K. \& Hightower, N. C. (I95I). Gastroenterology, 19, 480 .

\section{DISORDERS OF BOWEL FUNCTION IN SPINAL LESIONS}

\author{
By Dr. Volkmar Paeslack \\ Heidelberg, Germany \\ Spinal Centre, Orthopadic Unizersity Clinic
}

PARALYSIS of the bladder and bowels is expected in association with the diagnosis of paraplegia. Failure of the sympathetic nervous system is the part of the picture of spinal lesions which differentiates them from cerebral or peripheral nerve lesions. Although for this reason the urinary tract is automatically carefully watched nowadays, the bowel function is regarded as of less importance. Exact control of stomach and bowels is necessary if one wants to avoid severe complications during clinical treatment and for satisfactory social rehabilitation.

In every spinal lesion it is necessary to investigate the state of the digestive system. This will vary according to the level and extent of the lesion, the length of time the patient has been paralysed, the presence of complications, previous disorders, the age of the patient, and many other factors.

Certain special neuro-pathological points should be taken into consideration:

(I) The destruction of sympathetic innervation of the stomach and intestinal tract.

(2) The degree of paralysis of the abdominal muscles, the pelvic floor, and the external anal sphincter. 
The functions of these parts make up the action of abdominal compression, which is additionally influenced by the action of the diaphragm, the capacity of the patient to stand and sit, and absence or presence of spasticity of the skeletal muscle.

Further facts relevant in assessing bowel function are:

(a) Secretion and absorption by the mucous membrane of the stomach and bowels.

(b) The condition of bowel bacteria.

(c) The consistency of the bowel content, influenced by diet, water, the condition of ions, medicines, immobilisation or the degree of activity of the patient.

(d) The presence or absence of associated illness, complications such as general toxaemia, or disorder of the abdomen.

(e) Disease of the rectum, such as anal fissures, haemorrhoids, and anal prolapse, which actually produce a vicious circle.

These various factors influence the bowel function of individual patients in varying degrees.

Three main phases can be differentiated:

(I) The early phase, with functional failure due to spinal shock.

(2) A labile phase of bowel action in which automatic or autonomic action may gradually be introduced.

(3) A relatively stable condition resulting from careful treatment and exact training.

To understand the situation in these various stages of paraplegia, the most important neurological facts of bowel function must be remembered:

Voluntary cerebral control is required only for the taking in of food and for defaecation.

Everything else is the result of regulation of the intestinal organs by the sympathetic centres in the spinal cord, and in the tracts of the sympathetic nerves.

The parasympathetic innervation of the digestive tract as far as the transverse colon is concerned stems from the lower vagal centre.

The rectum has parasympathetic innervation from the pelvic plexus of the sacral cord.

The sympathetic control of the stomach and upper section of the intestine is by the coeliac ganglion, which receives its impulses mainly from the segments $\mathrm{T}_{5}$ to $\mathrm{L}_{3}$. These fibres, which are controlled from the sympathetic chain, reach the coeliac ganglion, and the superior mesenteric ganglion and the inferior mesenteric ganglion mainly through the splanchnic nerve. The sympathetic innervation works predominantly antagonistically, but also to a certain extent as a synergist to the parasympathetic impulses. It is important that these sympathetic, and partly also the parasympathetic fibres, next to the sympathetic control, conduct the afferent impulses to the spinal cord. Thus reflex arcs are set up which create a link between the bowels and the skin and skeletal musculature of the lower extremities, possibly also with unparalysed parts of the body. This fact has great significance in complete paraplegia.

Apart from the dominant action of these sympathetic and parasympathetic parts, digestion is controlled above all by the sensitive network of nerves situated 
in the stomach and bowel walls-the Meissner's and Auerbach's plexus. These sensitive organs guarantee the physiological development of bowel function in the higher lesions after the disappearance of spinal shock. In paraplegia the most severe disturbances are caused through failure of normal bowel evacuations. In high dorsal and cervical lesions, abdominal compression is absent. Cauda equina lesions produce severe disorders in so far as the parasympathetically innervated rectal muscle, the internal anal sphincter, and also the normal function of the external sphincter, are controlled by the somatic pudendal nerve.

Spinal shock produces at first full failure of the bowel-often also of the stomach function and at the same time atonia of the sphincter, especially in high dorsal and high cervical lesions. This results in atonic constipation, occasionally acute dilatation of the stomach, and an inclination towards abdominal distension. Rarely there is diarrhoea in the early phase which is very difficult to control.

Heavy accumulation of bowel contents and severe bowel distension considerably disturb the condition of the patient, especially in a new lesion. We find the abdomen distended, absence of bowel sounds, leading to vomiting, a raised temperature, and possibly to signs of ileus and acute dilatation of the stomach. In high lesions, especially in tetraplegia, this causes upward pressure on the diaphragm, which also interfere with breathing. It is therefore necessary to activate the bowel function, and to try to produce a satisfactory emptying from the earliest days.

Following the state of spinal shock, emptying of the bowels in high and dorsal lesions is achieved through action of the spinal reflex arcs. Thus the balance between the sympathetic and the parasympathetic nervous systems is gradually restored. At this time the patient must learn about the rhythmical tendencies of the sympathetic organs, and co-ordinate them with the remaining abdominal muscle function. This is the main factor in careful bowel training. In this phase of lability, diarrhoea occurs occasionally. The main problem for the patient, however, is constipation. Besides this failure of function, immobilisation and food deficient in cellulose (usually preferred by patients in bed) are contributory factors. Sedatives and analgesics no doubt add to the constipation.

It is important to diagnose the existing condition of the bowels. This demand, which is by no means easy to fulfil, is the basis of specific and successful treatment. We must therefore investigate whether the cause of constipation is a paralysis of all parts or only of a particular part of the bowel. One must try to discover whether disorders of secretion and absorption are playing a contributory part. A wellknown but frequently overlooked fact is the occurrence of protracted diarrhoea, with severe occlusive constipation in the caecum, transverse colon, or in even higher sections of the bowel. The thoughtless administration of antidiarrhoeic drugs can produce catastrophic results in these cases.

The diagnosis of the disorder of bowel function presents difficulties because of the lack of valuable subjective information from the patient. Nevertheless, with care, we can obtain useful information at the bedside. One begins with an examination of the whole patient. The most important observations concern oedema or signs of distension, the condition of the tongue, jaundice, or anaemia. Examination of the abdomen gives an idea of the distension, bowel rigidity, or hard faeces in a particular bowel section. Subsequent palpation of the abdomen determines the content of the individual bowel sections, especially the colon. Deep palpation may cause visceral pain in overstretched parts of the bowels, which 
is transferred and registered, as previously described, by the paraspinal tract. Auscultation of the abdomen in its various sections must not be neglected-the extent and character of the bowel sounds can be an important guiding sign.

The next step, the digital examination of the rectum, gives information about the state of the rectal contents, about the sphincter function, and about the existence of defects of the anal ring. If necessary one should do a proctoscopy or a rectoscopy, both of which are quick and simple methods of gaining important information about the condition of the rectum.

It is sometimes difficult to determine whether and to what extent the intestinal passages are slowed down or hindered. Oral administration of a marking substance (carbo medicinalis or small doses of X-ray contrast substance) and the registration of the passage-time are a simple clinical aid.

We can make further valuable deductions from an assessment of the faeces. It is therefore necessary to obtain a picture of the bulk, consistency, smell and colour. These examinations can be carried out with the help of diagnostic diets, which are combined with a coloured marking substance to determine the passage time. In certain cases the usual chemical and microscopic examinations of the faeces are additionally carried out. Occasionally, for example, after long-term administration of antibiotics, we order a bacteriological examination of the stools.

$\mathrm{X}$-ray examination of the empty abdominal organs shows the condition of the intestinal pathways, the bulk, and the possible existence and localisation of distension. The passage of orally administered contrast media, through the stomach and bowels and a contrast enema are rarely necessary, but may occasionally be required to provide information.

The synopsis of these various diagnostic measures gives information not only of the extent, localisation, and character of constipation, but also about the form of any possible diarrhoea, and the origin of pain. We also obtain a picture of any inflammation of the rectum, of haemorrhage in the digestive tract, of the functional potential of stomach and bowels. Especially in high lesions one must not overlook the investigation of the condition and mobility of the diaphragm, and the investigation of paroxysmal hypertension, which may originate from the overstretching of the bowels as well as from an increase in bladder pressure. In this connection one should also note the occasional incidence of critical abdominal spasms in high lesions. These are accompanied by considerable pain. The patient can not clearly define and localise them, but they have to be considered as a sign of near-ileus.

The treatment of constipation in paraplegia ought not to rely on single measures. Treatment must be extensive, and the many aspects of the facts mentioned must be considered.

The most important considerations are, firstly, the diet, and secondly the earliest possible activation of the patient. We advise a mixed diet, with good bulk, but avoiding flatulence-producing content. Peristalsis will be stimulated by fruit and fruit juices, sour milk, joghurt, and mineral water. Concentrated sugar extracts, particularly with lactose, have a good laxative effect.

Early and intensive physiotherapy serves not only the maintenance of muscle and joint function, the regulation of blood circulation and kidney function, but also supports the digestive activities. Regular turning of patients in the early phase is just as important as the general bodily activation after the disappearance of spinal shock. In severe cases of constipation it is possible that specific abdominal massage by an experienced hand may prove effective. In the early phase it may 
prove necessary to give small enemas as well as doing manual evacuation of the bowels.

Later one should attempt to break free from these methods. In most caudaequina lesions, anyway in those who have a dischezia, that is a complete paralysis of the intestines, such measures may be permanently necessary. In severe distension it is possible to bring relief to an exceptionally full intestinal tract, by giving digestive fermentations.

In the early phase, but also at the onset of secondary illnesses, the ileus and near-ileus produce increased difficulties from time to time. In such instances one should give varied doses of Prostigmin, and Pantothen acid, in combination, in carefully balanced infusions, for specific treatment. In rare cases it is necessary to drain the contents of the stomach and duodenum, and reintroduce sufficient fluids, by a stomach tube.

Very few paraplegics can manage for long without requiring laxatives. Boweltraining carefully carried out, can however reduce these drugs to a minimum. Drugs which act on the large intestine are preferable, such as aloe, senna, or acorus oil. Phenolphthalein and associated preparations have a similar action. Whereas these drugs have a special effect on the mucous membrane, we may in other cases obtain results by using laxative salts, such as those contained in mineral water, or with absorption-resistant sugar-alcohol.

The use of liquid paraffin should be limited to special cases because of the disorders of fat absorption.

The action of these various digestive remedies can be accompanied by small doses of so-called 'softeners' (Dioctil-Natrium-sulfosuccinat).

A clear understanding of the picture of the digestive functions presented in paraplegia, careful assessment of the existing state, and specific therapeutic methods make it possible to regulate existing disorders of function.

\title{
BOWEL TRAINING
}

\author{
By H. L. Frankel, M.B., M.R.C.P. \\ National Spinal Injuries Centre, Stoke Mandeville Hospital, Aylesbury
}

THE object of bowel training is regular and complete evacuation of the bowels a1 a chosen time and place, avoiding any leakage or bowel action in between, while at the same time avoiding constipation. The physiology of colonic, rectal and anal activity has been reviewed by Guttmann (1959), Connell, Frankel and Guttmann (1963) and Melzak and Porter (1964) and will not be described in this paper which deals with practical aspects of bowel management in patients with complete lesions of the spinal cord of cauda equina. Successful training is possible in almost every case if the doctor, nurse and patient understand the problems and know the methods of management available.

In the immediate stage after spinal cord injury there is often a period of slight or even absent activity of the small intestine; this ileus if recognised soon enough can be treated with regular injections of prostigmine during the first two or three days; we rarely need to pass a tube into the stomach and give intravenous therapy even in patients with cervical lesions and most patients can start a light diet by the third day; on the fourth and fifth day a mild laxative is given followed by 